\title{
The Persuasive Effect of Source Credibility: Tests of Cognitive Response
}

\author{
BRIAN STERNTHAL \\ RUBY DHOLAKIA \\ CLARK LEAVITT*
}

Two experiments are reported identifying the circumstances in which high credibility either facilitates, inhibits, or has no effect on the communicator's persuasiveness in relation to a less credible source. These data provide support for the cognitive response view of information processing and suggest the importance of message recipient's initial opinion as a determinant of persuasion.

$\mathrm{I}^{\mathrm{n}}$ experimental investigations of the persuasive effect of source credibility, it has been frequently demonstrated that highly trustworthy and expert spokespeople induce a greater positive attitude toward the position they advocate than do communicators with less credibility (cf. Sternthal, Phillips, and Dholakia in press). This finding can be explained in terms of cognitive response (cf. Greenwald 1968, 1970; Petty, Ostrom, and Brock 1978). According to this formulation, a message recipient's initial opinion is an important determinant of influence. In response to a persuasive appeal, individuals rehearse their issuerelevant thoughts, as well as those presented to them. Message rejection occurs when people opposed to the communicator's advocacy review counterarguments to assertions made to the message. If a highly credible source inhibits counterarguing, whereas a less credible source does not, cognitive response predicts the superior persuasive power of a highly credible communicator. Consistent with this interpretation, Cook (1969) reported less counterargumentation in response to a competent source than to an incompetent source.

Despite the substantial number of studies indicating that a highly credible source is more persuasive than a low credibility source, this finding is less than univocal.

- Brian Sternthal is Assistant Professor, Department of Marketing, Northwestern University, Evanston, IL 60201. Ruby Dholakia is Assistant Professor of Marketing, Indian Institute of Management, Calcutta, India. Clark Leavitt is Professor, Department of Marketing, Ohio State University, Columbus, OH 43210. The authors wish to thank Bobby Calder, Anthony Greenwald, Alice Tybout, and Peter Wright for their comments on drafts of this paper.
McGinnies (1973) reported that a highly credible source was more persuasive than a less credible communicator when a message recipient's initial opinion toward the advocacy was very negative, but no credibility effect emerged when they held a less negative initial opinion. Dholakia and Sternthal (1977) found no systematic credibility effect, even though the highly credible source was perceived to be more expert and trustworthy than the low credibility communicator. Dean, Austin, and Watts (1971) reported that their highly credible source induced greater persuasion when message recipients were adamantly opposed to the communicator's position, but the less credible source was more influential when the issue was one toward which individuals were likely to have had a favorable predisposition (e.g., counseling against an annual X-ray). Similarly, Bochner and Insko (1966) observed that a highly credible source was more persuasive than a moderately credible source when the advocacy was highly discrepant, but that the moderately credible source induced somewhat greater persuasion when the position advocated was relatively close to their initial opinion. Finally, Bock and Saine (1975) found that a low credibility source was more persuasive than a highly credible source when research participants favored the advocacy.

Although the majority of these investigations were not conceived as tests of cognitive response (an exception is Dholakia and Sternthal 1977), their findings can be interpreted in terms of this formulation. Specifically, the finding that a highly credible source exhibits greater persuasive power than one of lower credibility is consistent with the cognitive response 
prediction if it is assumed that message recipients had a negative predisposition toward the advocacy. In this situation, a credible source is more likely to inhibit counterargumentation than a source whose credibility is moderate or low. In turn, the reduction of counterargumentation stimulates persuasion.

The finding that a source who lacks credibility is more persuasive than a highly credible communicator is explained by cognitive response if it is assumed that individuals have a positive predisposition toward the message issue. In cognitive response terms, those favoring the advocacy will feel a greater need to insure that a position with which they agree is being adequately represented when the source's credibility is low than when the source's credibility is high. Therefore, it is expected that these individuals will generate more support arguments and will be more persuaded by a source lacking in credibility. The finding that credibility has no systematic persuasive effect is consistent with cognitive response if it is assumed that individuals had a neutral initial opinion toward the issue. In this case, they are likely to generate a similar number of support arguments and counterarguments in response to the appeal.

In sum, by making reasonable assumptions about a message recipient's initial opinions, cognitive response can order the persuasive main effect of credibility reported in previous investigations. A more compelling test of the cognitive response explanation of the credibility-persuasion relationship requires:

1. the systematic manipulation of source credibility,

2. a knowledge of the individual's initial opinion toward the communication issue, and

3. the measurement of thoughts as well as attitudes in response to an appeal.

The purpose of the present investigation was to provide such a test. To this end, two experiments were conducted. In Experiment I, the persuasive impact of a high and a moderate credibility source were examined in a context where research participants were likely to have a favorable initial opinion toward the message. In Experiment II, this test of cognitive response was extended by investigating the source credibility effect for subjects who had a negative initial opinion. The persuasive effect of credibility for all subjects, regardless of their initial opinion, was also examined in Experiment II.

This analysis was motivated by the desire to determine whether Dholakia and Sternthal's (1977) failure to observe a credibility main effect was attributable to the aggregation of responses from individuals varying in initial opinion within a credibility treatment. In both experiments, measures of attitudes and behavior as well as measures of support arguments and counterarguments were administered.

\section{EXPERIMENT I: METHOD}

\section{Overview}

Experiment I employed a $2 \times 2$ factorial design. Subjects who were likely to have a favorable initial opinion toward the issue were presented an appeal that was attributed to either a high or moderate credibility source. For half the subjects in each treatment the communicator was identified prior to the message, while for the remainder his identification was deferred until the end of the communication. After reading the appeal, its persuasive impact was measured on five indices: attitudes, intentions, support arguments, counterarguments, and behavioral response.

On the basis of cognitive response, it is hypothesized that the interaction between source credibility and timing of the source's identification will be significant. Specifically, it is expected that a moderately credible source will induce greater persuasion than a highly credible source when communicator identification precedes the message. As noted earlier, in this situation the moderately credible source is expected to stimulate greater support argumentation and, therefore, greater acceptance than the highly credible source. In contrast, when source identification follows the message, credibility cues are made available too late to affect the message recipient's thought generation process. As a result, it is predicted that the message will serve as the only influence cue, and source credibility will have no systematic persuasive effect.

The timing variable was chosen because it provides a way of determining the reliability of the credibility effects obtained in this experiment. Previous investigations have demonstrated that there is no systematic effect of credibility when source identification follows the message (cf. Ward and McGinnies 1974). If this finding is replicated, then confirmation of the cognitive response prediction that a source who lacks credibility will induce greater persuasion than a highly credible communicator when source identification precedes the message is unlikely to be due to some unknown factor peculiar to the present study.

\section{Issue}

Given the objective of the present research, the communication issue had to be one toward which message recipients evinced a positive initial disposition. At the same time, it was important that subject's predisposition was not so favorable as to preclude attitude change because of a ceiling effect. In a pretest, it was found that passage of the Consumer Protection Agency Bill (CPAB) fulfilled these criteria. Forty subjects, drawn from the same pool as those who participated in the main study, exhibited a favorable predisposition toward the bill on two 7-point Likert-type scales. Specifically, subjects indicated agreement with 
"freedom of private business results in the exploitation of consumers," $(\bar{X}=4.73, S . D .=1.39)$, and disagreement with "consumer interests are most effectively protected by business," $(\bar{X}=1.40, S . D$. $=.65$ ), two of the items employed in the main study to identify the attitudinal effects of the independent variables. For both these initial opinion items, mean attitudes were significantly more positive than the midpoint of the scale. ${ }^{1}$

\section{Subjects}

The 56 participants in this study were recruited from two sections of an undergraduate management course. During one of the regular class hours, the students were asked by their class instructor (who was not the experimenter) to participate in a study of federal legislation. Participation was voluntary. The sponsors of the study were described as an external group who had sought the assistance of the course instructor to administer the study. All the students agreed to participate.

The use of a convenience sample comprised of students is appropriate, given that the principal aim of the present research is to detect the relation between variables of theoretical significance. In such theory centered research, there are three requirements for external validity (Kruglanski 1975). First, the theoretical variables must be captured in the experimental setting (construct validity). Second, the operationalizations of the independent variables must be causally related to the operationalizations of the dependent variables (internal validity). Third, the causal relationships observed should not occur by chance (statistical conclusion validity). In contrast, those aspects of the situation that are theoretically irrelevant, in this instance sample representativeness, need not be considered. In fact. given that the present study entails theory-oriented research where individual differences are not of theoretical interest, between-subject variance is treated as random error that is appropriately kept to a minimum by selecting homogeneous samples (Kruglanski 1975). Therefore, the selection of a student sample for the present study in no way undermines the generation of inferences regarding the persuasive impact of source credibility or the adequacy of cognitive response in ordering the observed effects.

\section{Procedure}

Subjects were given booklets containing the independent and dependent variables by their instructor. The experimental task involved reading a one-page message dealing with the CPAB (S.707) that was pending before the United States Senate. The message pre-

\footnotetext{
${ }^{1} t=2.80, d f=38, p<.01$ for statement one and $t=21.67$, $d f=38, p<.001$ for statement two. All $t$ tests are one-tailed unless otherwise noted.
}

sented arguments in favor of the bill and stated the functions of the proposed agency. Depending upon the treatment to which subjects were assigned, the message was attributed to either a high or moderate credibility source, who was identified either before or after the message. These experimental conditions were administered in both classes to avoid confounding the effects of the independent variables. Once they had read the communication, subjects were asked to complete a questionnaire that included the dependent measures.

\section{Independent Variables}

Two independent variables were manipulated in the study: source credibility and timing of source identification. The source was described as a supporter of the bill and the reasons for his advocacy were given.

In the high credibility condition, the source was described as a Harvard-trained lawyer with extensive experience in the area of consumer issues and a recognized expert whose advice was widely sought. His support of this particular bill was made distinctive by describing him as a person who generally did not favor government controls, but who supported the $\mathrm{CPAB}$. The less credible source was portrayed as an individual with no special expertise, but one who was interested in consumer protection because of a job opportunity as consumer lobbyist. He was described as holding socialistic views with strong opinions in favor of all government controls.

The second independent variable involved manipulating the timing of the source's identification. For half the subjects, the source description appeared before the written communication, while for the remaining subjects the source description was deferred until the end of the communication.

\section{Measuring Instruments}

Five measures of persuasion, as well as a credibility manipulation check, served as the dependent variables. Message recipient's attitude toward the bill was determined first by administering the two attitudinal items used in the pretest, plus two items that focused more explicitly on the position advocated in the appeal: "Passage of the Consumer Protection Agency Bill would make me feel good" and "It is my belief that consumers do not need protection." Responses to these questions were measured on a seven-point scale ranging from strongly agree to strongly disagree. The four attitude questions were summed to form an overall score because they were highly interrelated (Cronbach $\alpha=.64$ ). These attitude measures were followed by a behavioral intention item where subjects indicated their likelihood of supporting the bill on a 100-point scale.

The next measure tapped message recipients' support arguments and counterarguments. Argumentation 
was determined by having subjects list all the thoughts that came to mind in response to the appeal. Three minutes were given to complete this task. Subjects then categorized their thoughts as either favorable or unfavorable to passage of the bill. For each subject, the thoughts listed that were consistent with the position taken in the message were summed to yield a support argument score, while the thoughts listed that opposed the message position were summed to yield a counterargument score.

The argumentation measurements were followed by two source evaluation measures. One measured subjects' perception of the trustworthiness and expertise of the source. Six items (three related to expertise and three related to trust), each rated on a sevenpoint semantic differential scale, were used for this purpose. Items included: expert - not expert; experienced - not experienced; trained-untrained; trustworthy - not trustworthy; moral-immoral; good-bad.

A second set of dependent variables which was not manipulated in the present study was related to attractiveness of the source. These nonequivalent source credibility items included: attractive-unattractive; dynamic-not dynamic; aggressive-not aggressive. These items are nonequivalent in the sense that unlike responses to the expertise and trust scales, responses to the attractiveness items should not be sensitive to the dependent variable. They were administered to determine whether demand characteristics accounted for the subject's perception of the source's credibility (Orne 1969). If the source credibility induction was effective and not attributable to demand characteristics, then subjects would perceive the highly credible source to be more trustworthy and expert than the moderately credible source, but they should not find one source more attractive than the other.

Behavioral compliance was determined by whether subjects signed a petition that was to be sent to their senators in Washington urging a vote for the bill.

\section{RESULTS}

\section{Manipulation Check}

A manipulation check determined whether the source credibility induction was effective. Subjects who received the message from the highly credible source perceived the communicator to be significantly more trustworthy and expert than did subjects who received the message from the moderately credible person. ${ }^{2}$ There was no difference between the high and moderate credibility sources on the nonequivalent attractiveness measures. ${ }^{3}$ Since the credibility manipulation involved

\footnotetext{
${ }^{2}$ High credibility: $\bar{X}=28.86, S \cdot D .=8.64, n=28$. Low credibility: $\bar{X}=20.57, S . D .=8.01, n=28, t=3.73, d f=54, p<.001$.

${ }^{3}$ High credibility: $\bar{X}=13.07, S . D .=4.52$. Low credibility: $\dot{X}$ $=13.68, S . D .=3.90$.
}

varying trustworthiness and expertise but not attractiveness, these data indicate that the credibility induction was effective and unlikely to be attributable to demand characteristics.

Although the means for the highly and moderately credible sources differ significantly on the trustworthiness and expertise dimensions, the moderately credible source was not perceived in a totally negative light. In fact, there was no difference between the moderately credible source and the scale midpoint $(t<1)$. Given that the experiment entailed having a source ask for compliance with a request to sign a petition, it was necessary that the less credible source have some favorable attributes. Indeed, in pretesting the credibility induction it was found that subjects perceived the situation to be contrived when the moderately credible source's biography was less favorable than the one used in this experiment.

\section{Attitudinal Response}

Subjects' attitudinal responses categorized by independent variables are reported in Table 1 . To determine the effects of the experimental variables on subjects' attitudes toward the CPAB, an analysis of variance was performed employing the sum of the attitudinal responses as the dependent measure. It was found that both source credibility $(F<1)$ and timing of the source's identification $(F=1.34, d f=1,52$, $p>.20$ ) did not have a significant effect on attitudes. As predicted, however, a significant disordinal interaction between source credibility and the time of the source identification was found $(F=15.97, d f=1,52$, $p<.001)$. The moderately credible source was more persuasive than the high credibility communicator when the credibility cue was presented before the message, whereas the highly credible source induced a more positive attitude when the credibility cue followed the communication as shown in the figure.

Although a significant source credibility $x$ timing interaction is necessary to confirm cognitive response predictions, it is not sufficient. It must also be demonstrated that:

1. the moderately credible source is significantly more persuasive than the highly credible source when the source credibility cues precede the message, and

2. the high and moderate credibility sources do not differ significantly in their persuasive power when the credibility cues appear after the message.

Using the Newman-Keuls procedure to make these contrasts, the above predictions were confirmed. When source identification preceded the message, the moderately credible source induced a more positive attitude than the highly credible source $(q=4.96, d f=52$, $p<.05$ ), whereas there was no systematic credibility 
TABLE 1

MEAN ( $\bar{X})$ AND STANDARD DEVIATIONS (S.D.) FOR ATTITUDES, SUPPORT ARGUMENTS, COUNTERARGUMENTS, AND INTENTIONS CATEGORIZED BY INDEPENDENT VARIABLES, SOURCE CREDIBILITY, AND TIMING OF THE CREDIBILITY INDUCTION

\begin{tabular}{|c|c|c|c|c|c|}
\hline \multirow{3}{*}{$\begin{array}{l}\text { Timing of the } \\
\text { credibility induction }\end{array}$} & \multirow[b]{3}{*}{$\begin{array}{l}\text { Dependent } \\
\text { measure }\end{array}$} & \multicolumn{4}{|c|}{ Source credibility } \\
\hline & & \multicolumn{2}{|c|}{ High } & \multicolumn{2}{|c|}{ Moderate } \\
\hline & & $\dot{x}$ & S.D. & $\bar{x}$ & S.D. \\
\hline Source before message (SM) & $\begin{array}{l}\text { Attitude } \\
\text { Support arguments } \\
\text { Counterarguments } \\
\text { Intention }\end{array}$ & $\begin{array}{r}19.64 \\
0.71 \\
1.43 \\
62.14\end{array}$ & $\begin{array}{r}5.17 \\
1.20 \\
1.34 \\
28.06\end{array}$ & $\begin{array}{r}25.00 \\
1.86 \\
0.93 \\
89.00\end{array}$ & $\begin{array}{r}3.04 \\
1.92 \\
1.00 \\
11.90\end{array}$ \\
\hline Source after message (MS) & $\begin{array}{l}\text { Attitude } \\
\text { Support arguments } \\
\text { Counterarguments } \\
\text { Intention }\end{array}$ & $\begin{array}{r}22.71 \\
1.29 \\
0.79 \\
78.29\end{array}$ & $\begin{array}{r}2.89 \\
1.82 \\
1.05 \\
15.23\end{array}$ & $\begin{array}{r}19.43 \\
0.93 \\
1.57 \\
59.64\end{array}$ & $\begin{array}{r}4.60 \\
1.21 \\
1.87 \\
27.16\end{array}$ \\
\hline
\end{tabular}

effect when the source identification was deferred until after the message ( $q=2.97, d f=52, p>.05)$. Furthermore, the high credibility source induced a significantly more positive attitude toward the bill when the source was identified after-rather than before the message $(q=2.78, d f=52, p=.05)$. However, the moderately credible source was significantly more influential when introduced before the message ( $q$ $=5.15, d f=52, P<.01)$.

\section{Argumentation-Counterargumentation}

A more compelling test of cognitive response requires operationalizing the variables presumed to medi-

\section{FIGURE}

SOURCE CREDIBILITY $\times$ TIMING OF CREDIBILITY INDUCTION

\section{Attitude}

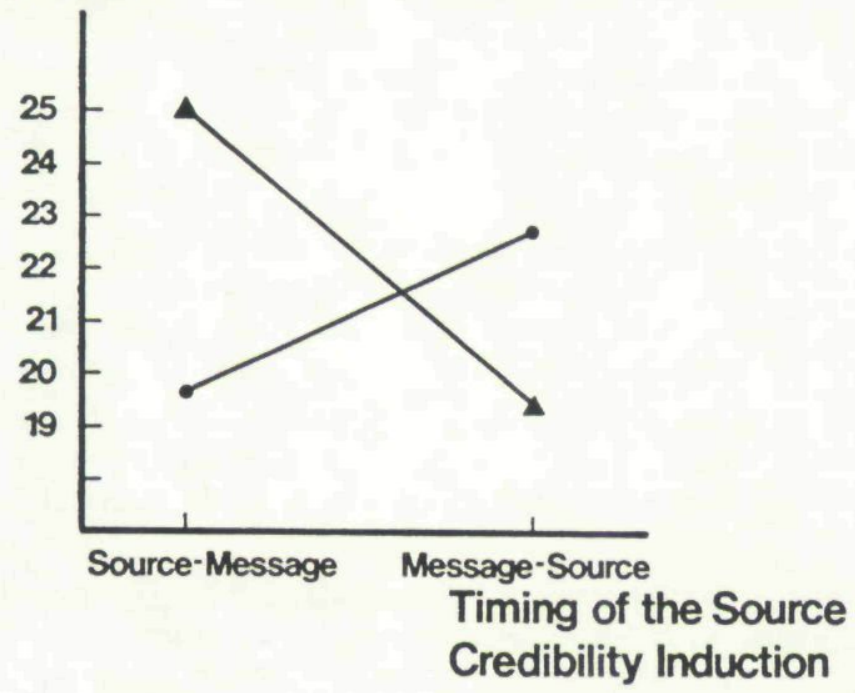

- -High Credibility Source

$\Delta$-Moderate Credibility Source ate persuasion - support argumentation and counterargumentation. To address this issue, the effects on argumentation of source credibility and timing of the source identification were examined (See Table 1). The source credibility $x$ timing of source identification was marginally significant for support argumentation $(F=3.19, d f=1,52, p=.08)$ and counterargumentation $(F=3.13, d f=1,52, p=.08)$. As predicted, when source identification preceded the message, subjects generated more support arguments in response to the moderately credible source than the highly credible one $(t=2.74, d f=54, p<.01)$. Also congenial to the cognitive response formulation was the finding of no differences in support argumentation attributable to source credibility when source identification occurred after the message $(t<1)$ and the observation of no credibility effects on counterargumentation (identification before message, $t=1.58$, $d f=54, p>.10$; identification after message, $t$ $=1.95, d f=54, p>.05){ }^{4}$

To evaluate the mediating role of support arguments and counterarguments further, the effect of the treatments on attitude was determined when the number of each of these thought types was treated as a covariate. When the number of support arguments served as a covariate, the independent variables still had a significant effect on attitudes $(F=4.62$, df $=3,51, p<.01$ ). Nevertheless, support arguments accounted for a substantial and statistically significant portion (38 percent) of the explained attitude variance $(F=3.63, d f=3,51, p<.05)$. Similarly, when the number of counterarguments was treated as a covariate, the treatment-attitude relationship was maintained $(F=5.01, d f=3.51, p<.005)$, though counterarguments accounted for a significant portion of the explained variance $(F=3.87, d f=3,51, p<.05)$.

\footnotetext{
4 Two-tailed tests were used in making these contrasts, because no source credibility effects were predicted from cognitive response.
} 


\section{Intention and Behavior}

Analysis of effects on behavioral intention yielded essentially the same results as just reported for attitudes. An analysis of variance indicated that only the source credibility $x$ timing interaction was significant $(F=15.27, d f=1,52, p<.001)$. Systematic comparison of treatments using the Newman-Keuls procedure revealed that the significant interaction was attributable to the moderately credible sources inducing a significantly greater intention to support the bill than the highly credible source when the credibility cues preceded the message $(q=4.62, d f=52, p<.01)$. When the source's identification followed the message, there was no systematic effect of source credibility on subjects' behavioral intentions $(q=3.20, d f=52, p$ $>$.05).

Finally, neither source credibility nor the timing of the source credibility induction had a significant effect on subjects' behavioral responses $\left(\chi^{2}=3.64\right.$, $d f=3, p>.30)$. However, the behavioral data exhibit a pattern similar to that obtained for attitudinal and intention responses. Subjects complied more with the moderately credible source when the source induction preceded the message, whereas the highly credible source induced greater compliance when the source induction was deferred until after the message.

\section{EXPERIMENT II: METHOD}

The results of Experiment I provide support for the cognitive response formulation where message recipients were likely to have been favorably predisposed to the communication issue. To provide a stronger test of cognitive response predictions, the effect of source credibility upon favorably and negatively predisposed individuals was investigated in Experiment II. Subjects were drawn from the pool used in Experiment I. The question battery included five Likert-type scales pertaining to participants' attitudes toward the issue. ${ }^{5}$ Subjects' responses on these five scales were summed to yield an initial opinion score. Subjects whose score was at or above the median were classified as having a "positive initial opinion" toward the issue; those with scores below the median were classified as having a "negative initial opinion." 6

Approximately five weeks later, a second experimenter requested that subjects participate in a study to determine individuals' feelings about the CPAB.

\footnotetext{
s The scales included: "It is more important to enforce the consumer protection legislation that is already on the books than to pass more laws to protect consumers." "It is my belief that consumers do not need protection. "'.Consumers' interests are most effectively protected by business." "Freedom of private business from government regulation results in exploitation of consumers." "The only way to ensure that consumers are not exploited is to pass laws protecting consumers." Subjects responded to each statement on a five-point scale.

"A "neutral" initial opinion group was not established because it would have made cell sizes very small.
}

The 37 participants followed essentially the procedure used in Experiment I, although the timing of the source's identification was not manipulated in Experiment II. Subjects read a communication attributed to either a highly or moderately credible source. The credibility induction appeared at the outset of the message. Then, the dependent measures were administered. First, the subjects completed the thought sampling question by listing and rating their support arguments and counterarguments relevant to passage of the CPAB. Three minutes were given to complete this task. Next, subjects rated their attitudes toward the bill on ten 7-point Likert-type scales. Five of these scales were the same as those administered in the pretest, while the remaining five were specific to the bill. ${ }^{7}$ This was followed by the nine 7-point semantic differential scales pertaining to the source's attributes of character used in Experiment I. Finally, subjects completed the request to sign the petition by signing it or indicating their refusal to support the bill.

The dependent variable strategy employed in Experiment II departs from that used in Experiment I in several ways. The number of attitude scales were increased in Experiment II to ensure the reliability of the attitude measure $(\alpha=.78)$. In addition, the order in which the dependent variables were administered was altered. Rather than have subjects complete the attitude scales prior to administering the thoughts measure, as was the case in Experiment I, subjects' support arguments and counterarguments were elicited before their attitudes were measured in Experiment II. This change eliminated the possibility that responses on the scaled attitude measures would affect the support arguments and counterarguments (Wright 1977).

On the basis of cognitive response, two predictions were made. It was hypothesized that subjects with a positive disposition toward the communication issue would be more persuaded by the moderately credible source than by the high credibility source, replicating the result found in Experiment I. In contrast, for individuals who were negatively predisposed to the issue, a highly credible source was expected to be more persuasive.

\section{RESULTS}

\section{Manipulation Check}

To check the source credibility induction, the ratings given on the trust and expertise items by subjects assigned to the high credibility condition were summed and compared with the summed ratings

"The attitude scales specific to the bill included: "Passage of the Consumer Protection Agency Bill (CPAB) would make me feel good." "I feel very negatively toward the CPAB." "I would personally support establishing the CPAB." "It is my belief that the CPAB merits public support." "If asked, I would not be willing to give my time to ensure passage of the CPAB." 
TABLE 2

MEAN ( $\bar{X})$ AND STANDARD DEVIATIONS (S.D.) FOR ATTITUDES, SUPPORT ARGUMENTS, AND COUNTERARGUMENTS CATEGORIZED BY SOURCE CREDIBILITY TREATMENT AND TYPE OF SAMPLE

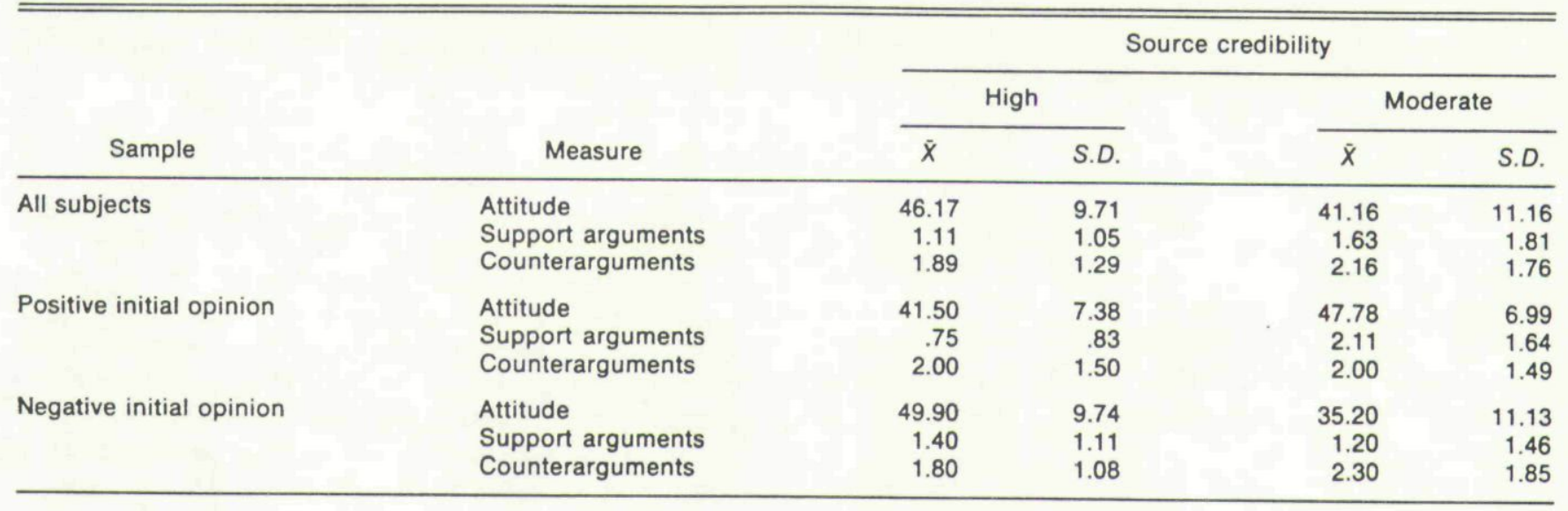

given by subjects assigned to the moderate credibility condition. It was found that the highly credible source $(\bar{X}=30.78, S . D .=5.21, n=18)$ was perceived to be more trustworthy and expert than the moderately credible communicator $(\bar{X}=20.47, S . D .=4.29, n$ $=19 ; t=6.57, d f=35, p<.01)$. No treatment differences were obtained with respect to the nonequivalent attractiveness measures. ${ }^{8}$ The source credibility induction was also successful for the subset of the message recipients who had a positive initial opinion toward the issue ${ }^{9}$ and who had a negative initial opinion. ${ }^{10}$

\section{Effect of Source Credibility}

The data analysis involved the responses of the entire sample, those with a positive initial opinion toward the issue and those with a negative initial opinion. The means and standard deviations of the dependent variables for each of these groups, categorized by the independent variable, are shown in Table 2. Analysis of the mean attitudinal responses obtained for the entire sample indicated that the highly credible source induced a more positive attitude toward the CPAB than did the moderately credible source, though this difference did not reach statistical significance $(t=1.46, d f=35, p>.05)$. This finding replicates the result obtained in an experiment using a similar paradigm reported by Dholakia and Sternthal (1977). Furthermore, source credibility did not have a significant effect upon support argument $(t=1.08$, $d f=35, p>.20)$ or counterargument production

\footnotetext{
${ }^{8}$ High credibility, $\tilde{X}=12.79$, S.D. $=2.94$ : low credibility $\tilde{X}$ $=11.50, S . D .=1.95 ; t=1.59, d f=35, p>.05$

${ }^{2}$ High credibility $\dot{X}=29, S . D .=4.61, n=8$; low credibility $\bar{X}=21 . S . D .=2.90, n=9 ; t=4.23, d f=15, p<.01$.

10. High credibility $\bar{X}=32.20, S . D .=5.15, n=10$; low credibility $\bar{X}=20, S . D .=5.20, n=10 ; t=5.28, d f=18, p<.01$.
}

$(t<1)$. Also the communicator's credibility did not affect compliance with the request to sign the petition favoring the $\mathrm{CPAB} ; 28$ percent of those receiving the message from the highly credible source signed the petition and 32 percent of those receiving the appeal from the moderately credible source signed.

Examination of the impact of source credibility for subjects with a relatively positive initial opinion toward the communication issue (Table 2 ) indicates that the moderately credible source induced a more positive attitude toward the issue than the highly credible communicator $(t=1.79, d f=15, p<.05)$. Furthermore, those who received a message attributed to the moderately credible source generated more support arguments than did subjects for whom it was attributed to a highly credible source $(t=2.19, d f=15, p<.025)$. When the number of support arguments were treated as a covariate, the effect of source credibility on attitudes was not significant $(F=.84, d f=1,14, p>.35)$, In contrast, source credibility had no systematic effect on the generation of counterarguments $(t<1)$, and treating the number of counterarguments as a covariate did not have a substantial effect on the credibility-attitude relationship $(F=6.88, d f=1,14, p$ $=.02$ ). Finally, the source's credibility did not have a significant influence on subject's response to the request to sign the petition $\left(\chi^{2}=1.55, d f=1, p>.20\right)$, although compliance was somewhat greater in response to the moderately credible source ( 56 percent) than to the highly credible communicator ( 25 percent). These findings essentially replicate the results reported for the treatment where source identification preceded the message in Experiment $\mathrm{I}$.

These analyses were repeated for message recipients who had a relatively negative initial opinion toward the communication issue. Consistent with the findings reported in most previous credibility main effect studies, the highly credible source induced a more 
positive attitude toward his advocacy than did the less credible communicator $(t=3.14, d f=18, p<.01)$. However, source credibility did not have a systematic effect on either counterargument $(t<1)$ or support argument $(t<1)$ generation. And, treating the number of thoughts as a covariate did not affect the credibility-attitude relationship which was significant whether support arguments $(F=8.53, d f=1,17$, $p=.01)$ or counterarguments $(F=9.35, d f=1,17$, $p<.01)$ served as the covariate. Furthermore, credibility did not systematically affect compliance $\left(\chi^{2}\right.$ $=1.08, d f=1, p>.20$ ), although those receiving the communication from the highly credible source showed a greater tendency to sign the petition than those who received it from the less credible source (30 percent versus 10 percent).

\section{DISCUSSION}

Three major findings emerge from these experiments. First, when message recipients were favorably predisposed to an issue and the communicator was identified prior to the message, the moderately credible source induced more agreement and support argumentation than did the highly credible communicator. This result was obtained in both Experiments I and II. Second, when the source was identified after the message, credibility had no systematic effect on attitudes, support argumentation, or counterargumentation (Experiment I). Third, when message recipients were negatively predisposed to the communication issue, the highly credible source induced more agreement than the less credible communicator (Experiment II), though source credibility did not affect thought generation.

These results generally support the cognitive response predictions. In cognitive response terms, the moderate credibility source induced greater positive attitude and support argumentation when identified at the outset of the communication, presumably because message recipients felt a need to bolster support for a position they favored when the communicator was of questionable credibility. They felt less inclined to engage in this cognitive work when a highly credible source was presenting a favored position. In contrast, there was no source credibility effect when source identification followed the message because the credibility cue was made available too late to mediate the thought generation process.

The failure to observe a significant effect of source credibility on counterargumentation in both of the timing conditions examined in Experiment $\mathrm{I}$ is predicted by the cognitive response position. Because subjects had a favorable disposition toward the issue, it was anticipated that the source credibility cue would have its predominant effect on support argumentation rather than counterargumentation. The superior persuasive power of the highly credible source when message recipients were initially opposed to the advocacy is also predicted by cognitive response. In this situation, a highly credible source serves to inhibit counterargumentation, whereas a less credible source facilitates it.

Also of interest is the finding (Experiment II) that source credibility has a systematic effect on thought generation and on attitudes when subjects are categorized by their initial opinion, but not when the responses of these groups are aggregated. This result may explain the failure to observe a source credibility effect in a previous investigation conducted by Dholakia and Sternthal (1977) using a paradigm similar to that in the present study. By aggregating the responses from subjects differing in initial opinion, Dholakia and Sternthal may have washed out the credibility effect.

Despite the general support for the cognitive response formation, several findings are at odds with the predictions made from this theory. One pertains to the effect of the independent variables on message recipient's compliance with the request to sign the petition favoring the bill. Although the compliance data were in the same direction as the other measures of persuasion in both experiments, source credibility did not have a statistically significant effect on an individual's behavioral response. This result is not only inconsistent with the cognitive response prediction, but it questions the importance of the source credibility variable when consumer's behavioral response is of focal concern. However, before it is concluded that source credibility does not have a systematic effect on behavior, or that cognitive response does not order this effect, the negative finding must be demonstrated in situations where the communicator's attributes are highly salient to message recipients. The present investigation does not provide such a test. Salience of the source credibility may be achieved by presenting the communicator in person. Indeed, when this approach was used, source credibility did have a significant effect on behavior (Brock 1965; Woodside and Davenport 1976).

The finding (Experiment II) that individuals exposed to the moderately credible source who were negatively predisposed to the issue did not generate significantly more counterarguments than those receiving the message from the highly credible source is also inconsistent with cognitive response. This result may be attributable to the fact that subjects in the moderate credibility-negative initial opinion condition were unable to generate a substantial number of counterarguments because of their lack of familiarity with the issue. Thus, while they exhibited a less favorable attitude toward the bill when source credibility was moderate, they were unable to verbalize many thoughts related to that disposition.

Finally, although the credibility treatment has a substantial effect on the thoughts generated by subjects in Experiment I, contrary to the cognitive response 
prediction, treating thoughts as a covariate still resulted in a significant credibility-attitude relationship. This finding may be attributable to thought samples being affected by the attitude and intention dependent measures which preceded them. Support for this contention emerges from the analysis of responses from those who were favorably predisposed to the message issue in Experiment II. In this instance, thought samples were generated before other dependent measures. When this procedure was followed, treating support arguments as a covariate yielded a nonsignificant source credibility effect on attitude, as predicted by cognitive response. This finding suggests that thought sampling measures should be administered prior to other dependent measures in future tests of cognitive response (cf. Wright 1977).

[Received February 1977. Revised September 1977.]

\section{REFERENCES}

Bochner, Stephen, and Insko, Chester (1966), " Communication Discrepancy, Source Credibility, and Opinion Change," Journal of Personality and Social Psychology, 4, 614-21.

Bock, Donald, and Saine, Thomas (1975), "The Impact of Source Credibility, Attitude Valence, and Task Sensitization on Trait Errors in Speech Evaluation," Speech Monographs, 37, 342-58.

Brock, Timothy (1965), "Communicator-Recipient Similarity and Decision Change," Journal of Personality and Social Psychology, 1, 650-4.

Cook, Thomas (1969), " Competence, Counterarguing, and Attitude Change," Journal of Personality, 37, 342-58.

Dean, Robert, Austin, John, and Watts, William (1971), "Forewarning Effects in Persuasion: Field and Class- room Experiments," Journal of Personality and Social Psychology, 18, 210-21.

Dholakia, Ruby and Sternthal, Brian (1977), "Highly Credible Sources: Persuasive Facilitators or Persuasive Liabilities?" The Journal of Consumer Research, 3, 223-32.

Greenwald, Anthony (1968), "Cognitive Learning, Cognitive Response to Persuasion, and Attitude Change," in Psychological Foundations of Attitudes, eds. Anthony Greenwald, Timothy Brock, and Thomas Ostrom, New York: Academic Press, 147-70.

Greenwald, Anthony (1970), "Initial Opinion and Response to Persuasion," proposal to the National Science Foundation.

Kruglanski, Arie (1975), "The Two Meanings of External Validity," Human Relations, 28, 653-59.

McGinnies, Elliot (1973), "Initial Attitude, Source Credibility, and Involvement as Factors in Persuasion," Journal of Experimental Social Psychology, 9, 285-96.

Orne, Martin (1969), "Demand Characteristics and the Concept of Quasi-Controls," in Artifact in Behavioral Research, eds. R. Rosenthal and R. Rosnow, New York: Academic Press.

Petty, Roger, Ostrom, Thomas, and Brock, Timothy (1978), Cognitive Responses in Persuasive Communication: A Text in Attitude Change, New York: McGraw Hill.

Sternthal, Brian, Phillips, Lynn, and Dholakia, Ruby (in press), "The Persuasive Effect of Source Credibility: A Situational Analysis," Public Opinion Quarterly.

Ward, Charles, and McGinnies, Elliot (1974), "Persuasive Effects of Early and Late Mention of Credible and Noncredible Sources," Journal of Psychology, 86, 17-23.

Woodside, Arch and Davenport, James (1976), "Effects of Price and Salesman Expertise on Customer Purchasing Behavior," The Journal of Business, 49, 51-9.

Wright, Peter (1977), "'Research on Ad-Stimulated Thought Processes: A Review," Faculty Working Paper, Graduate School of Business. Stanford University. 
Copyright of Journal of Consumer Research is the property of Journal of Consumer Research. Inc. and its content may not be copied or emailed to multiple sites or posted to a listserv without the copyright holder's express written permission. However, users may print, download, or email articles for individual use. 